schädigung gewertet werden kann. Die Zellmembran hält im Bereiche der Tropfen dem offenbar gesteigerten Binnendruck lange stand und platzt erstaunlicherweise auch nach Erreichen erheblicher Größe der Tropfen nicht. Sie schließt sich vielmehr infolge ihrer Elastizität bei der Ablösung der Tropfen (Ptyse), so daß weder bei letzteren, noch beim Zellrest lichtmikroskopisch faßbare Verluste der Zellmembran auftreten. Über die elektronenmikrosko- pischen Untersuchungen zusammen mit PROPST 22 wird an anderer Stelle ausführlich berichtet werden.

Für die teilweise Subventionierung dieser Arbeit ist das Pathologisch-anatomische Institut der Firma Hoffmann-La Roche, Wien, zu Dank verpflichtet.

22 A. Propst, M. Ratzenhofer u. J. ZangGer (in Vorbereitung).

\title{
Inaktivierungsversudhe mit homozygoten Hefestämmen verschiedenen Ploidiegrades
}

\author{
VII. Vergleichende Untersuchung Strahlen- und Bernsteinsäureperoxyd-induzierter \\ Atmungs-, Gärungs- und Knospungshemmungen \\ Von Sigrid Hempel * und Wolfgang Laskowski \\ Aus dem Institut für Biophysik der Freien Universität Berlin
}

(Z. Naturforschg. 19 b, 929-935 [1964] ; eingegangen am 4. Juni 1964)

\begin{abstract}
A diploid Saccaromyces strain was treated with several doses of X-rays, UV and succinic acid peroxyde (BPO). The inactivation of the ability to form macroscopic colonies as well as the ability to form microcolonies of at least two cells to a few hundred cells has been compared with the inactivation of respiration and fermentation intensity. If the inactivation of macroscopic colony formation is taken as a measure of the effective dose applied, the formation of at least one daughter cell as well as respiration and fermentation intensity is reduced to approximately the same extent after BPO and X-ray treatment. In the latter case, however, much higher effective doses have to be applied and a distinct difference between respiration and fermentation sensitivity is observed. After UV-treatment the formation of at least one daughter cell is exceedingly more sensitive than the fermentation processes. The respiration processes behave most UV resistant. Possible reasons for the observed different relative sensitivities are discussed.
\end{abstract}

Werden Hefezellen zunehmenden Dosen ionisierender und nicht ionisierender Strahlen ausgesetzt, so kommt es u. a. zu einer zunehmenden Inaktivierung des Koloniebildungs-Vermögens. Abhängig von der absorbierten Strahlendosis, dem Ploidiegrad und bestimmten Stoffwechselzuständen der Zellpopulation ist ein Teil der Zellen in der Lage, nach mehrtägiger Bebrütung auf Vollmedium makroskopische Kolonien zu bilden. Bei den übrigen nicht makroskopische Kolonien bildenden Zellen ist das Knospungsvermögen entweder sofort völlig gehemmt, die Zellen bleiben als Einzelzellen liegen, oder aber es erlischt nach der Bildung einiger Tochterzellen, und Mikroklone von 2 bis zu einigen

* Jetzige Anschrift: Institut für Gärungsgewerbe, Berlin 65.

1 W. Laskowski u. W. Stein, Z. Naturforschg. 13 b, 305 [1958]. hundert Zellen werden gebildet ${ }^{1}$. Bezogen auf den Anteil makroskopisch Überlebender zeigt die Häufigkeit strahleninduzierter Einzelzellen und Mikroklone (Restteilungsmuster) bestimmte Unterschiede in Abhängigkeit von Ploidiegrad und Inaktivierungsnoxe ${ }^{2}$. Eine absolute Knospungshemmung der Mehrzahl der behandelten Zellen tritt aber allgemein erst nach Anwendung recht hoher Strahlendosen auf. Da verschiedene Beobachtungen vorliegen ${ }^{3}$, daß in derart hohen Dosisbereichen bereits wesentliche Stoffwechselprozesse wie Atmung oder Gärung merklich gehemmt werden, wurde in l.c. ${ }^{2}$ die Hypothese aufgestellt, daß die strahleninduzierte absolute Knospungshemmung mit einer Hem-

2 W. Laskowski u. W. Stein, Z. Naturforschg. 15 b, 604 [1960].

3 Übersich bei A. Rothstein, Radiat. Res. Suppl. 1, 357 [1959]. 
mung der Atmungs- und/oder Gärungsprozesse korreliert sein könnte. Um diese Arbeitshypothese zu überprüfen, wurde eine vergleichende Untersuchung der Knospungs-, Atmungs- und Gärungshemmung nach Behandlung eines diploiden Hefestammes mit ultraviolettem Licht und Röntgenstrahlen vorgenommen, über deren Ergebnisse hier berichtet werden soll. Da außerdem frühere Beobachtungen ${ }^{1,2}$ gezeigt hatten, daß durch organische Peroxyde ein grundsätzlich andersartiges Restteilungsmuster als durch Strahlen hervorgerufen wird - es treten praktisch keine Mikroklone, sondern alternativ Einzelzellen bzw. makroskopische Kolonien auf - wurden die Folgen einer Behandlung mit Bernsteinsäureperoxyd ebenfalls untersucht.

\section{Material und Methode}

\section{Inaktivierungsversuche}

Alle Versuche wurden mit dem diploiden Saccharo. myces-Stamm 211 durchgeführt, dessen Herkunft bereits beschrieben wurde ${ }^{4}$. Für die Versuche wurde jeweils eine Probe des Stammes in $5 \mathrm{ml}$ Medium, bestehend aus $1 \%$ Hefeextrakt und $0,25 \%$ Glucose, geimpft und für $48 \mathrm{Stdn}$. bei $30{ }^{\circ} \mathrm{C}$ ohne zusätzliche Belüftung bebrütet. Nach $48 \mathrm{Stdn}$. war die Kultur in der stationären Wachstumsphase und enthielt weniger als $10 \%$ Zellen mit Knospen. Nach 3-maligem Waschen in $m / 20-\mathrm{KH}_{2} \mathrm{PO}_{4}$-Puffer (im folgenden "Puffer" genannt) wurden die Zellen auf einen Titer von $2,5 \cdot 10^{8}$ oder $6 \cdot 10^{7}$ Zellen pro ml Puffer eingestellt. Für die Bestrahlungsversuche wurde jeweils $1 \mathrm{ml}$ dieser Zellsuspension in ein Glasschälchen pipettiert (Schichthöhe etwa $2 \mathrm{~mm})$. Die Röntgenstrahlen-Experimente wurden mit einer Machlett-Röhre (Typ OEG-60, W-Anode, Be-Fenster) ohne zusätzliche Filterung bei $50 \mathrm{kV}$ Röhrenspannung durchgeführt. Bei einem Focusabstand von $15 \mathrm{~cm}$ und $9 \mathrm{~mA}$ Röhrenstrom betrug die Dosisleistung $\approx 10 \mathrm{kr} /$ Minute. Zur UV-Bestrahlung diente eine $\mathrm{Hg}$ Niederdrucklampe (Osram HNS 12), die etwa 90\% ihrer Intensität mit der Wellenlänge $254 \mathrm{~nm}$ ausstrahlt. Die Strahlenintensität betrug $1,22 \cdot 10^{3} \mathrm{erg} / \mathrm{cm}^{2} \mathrm{sec}$. Die Hefesuspensionen wurden während der Bestrahlung mit einem magnetischen Rührer ständig durchmischt. Zur Behandlung mit Bernsteinsäureperoxyd (BPO) wurden Zellsuspensionen (Titer $2,5 \cdot 10^{8}$ Zellen $/ \mathrm{ml}$ ) BPO-Lösungen verschiedener Konzentration für jeweils $30 \mathrm{~min}$ ausgesetzt und währenddessen magnetich gerührt. Darauf wurden die Zellen abzentrifugiert und 3-mal mit Puffer gewaschen.

Zur Bestimmung der Inaktivierungsraten wurden die behandelten Zellen sofort nach Abschluß der Behandlung in geeigneter Weise verdünnt, auf Nähragarplatten (1\% Hefeextrakt, $0,5 \%$ Pepton, $2 \%$ Glucose, $2 \%$

4 W. Laskowski, Z. Naturforschg. 15 b, 495 [1960].
Agar) ausgespatelt und 3 bis 4 Tage bei $30{ }^{\circ} \mathrm{C}$ bebrütet. Mit unbehandelten Zellen wurde - bis auf die entsprechende Behandlung - gleichartig verfahren.

Die mikroskopische Bestimmung der Anteile inaktiver Einzelzellen und Mikroklone wurde bereits beschrieben ${ }^{1}$. Die Zellknospung wurde in der Regel nach $3,7,11,24,48$ und $72 \mathrm{Stdn}$. Bebrütung bei $30^{\circ} \mathrm{C}$ verfolgt. Nach 48 Stdn. war im allgemeinen das Restteilungsmuster festgelegt. Zellen, die bis dahin keine Knospe aufwiesen, blieben als Einzelzellen liegen.

\section{Messung der $\mathrm{O}_{2}$-Aufnahme}

Die Messungen wurden mit einem W a r b u r g - Apparat (Typ SL 85) durchgeführt. Je $1 \mathrm{ml}$ der gewaschenen und in Puffer suspendierten Zellen (Titer $2,5 \cdot 10^{8}$ Zellen $/ \mathrm{ml}$ ) wurde in ein Reaktionsgefäß (Typ A $05-00)$ eingefüllt. Der Gefäßeinsatz enthielt $0,3 \mathrm{ml}$ einer 20-proz. KOH-Lösung und die Ansatzbirne 0,2 ml einer 12-proz. Glucoselösung. Die Temperatur des Wasserbades betrug $30{ }^{\circ} \mathrm{C}$, die Schüttelfrequenz der Manometer 100 Ausschläge/min bei einer Amplitude von $2 \mathrm{~cm}$. Nach einer Eintemperierungszeit von $15 \mathrm{~min}$ wurden die Gefäße geschlossen, die Glucoselösung zugegeben und die Druckänderungen in Abständen von 10 min über einen Zeitraum von $60 \mathrm{~min}$ abgelesen und auf $\mathrm{O}_{2}$. Verbrauch umgerechnet.

\section{Messung der $\mathrm{CO}_{2}$-Abgabe}

Die Messungen wurden mit dem gleichen Gerät wie die $\mathrm{O}_{2}$-Aufnahme durchgeführt. Der Zelltiter betrug $6 \cdot 10^{7}$ Zellen $/ \mathrm{ml}$ Puffer. Je $1 \mathrm{ml}$ Hefesuspension wurde in ein Gefäß (Typ A 05-00) eingeimpft. In die Ansatzbirne wurde $0,2 \mathrm{ml}$ einer 12-proz. Glucoselösung gegeben. Um anaerobe Bedingungen zu erhalten, wurden vor Beginn der Messung für 15 min hochgereinigter Stickstoff aus einer Bombe der Firma Linde $(99,98 \%$ $\mathrm{N}_{2}$ ) durch die Gefäße geleitet. Die Daten für Wasserbadtemperatur, Schüttelfrequenz und -amplitude entsprechen denen bei der Messung des $\mathrm{O}_{2}$-Verbrauches. Nach Zugabe der Glucoselösung wurden die Druckänderungen in Abständen von 10 min über einen Zeitraum von $60 \mathrm{~min}$ abgelesen und auf $\mathrm{CO}_{2}$-Abgabe umgerechnet.

In den Bestrahlungsversuchen wurden die bestrahlten Zellsuspensionen unmittelbar nach der Bestrahlung in die Reaktionsgefäße überführt. Etwa 30 min verstrichen zwischen Bestrahlungsende und Beginn der manometrischen Messung. In den BPO-Experimenten wurden nach $30 \mathrm{~min}$ BPO-Einwirkungszeit die Hefezellen abzentrifugiert, 3-mal gewaschen, in Puffer resuspendiert und in die Reaktionsgefäße übertragen.

\section{Ergebnisse}

Die $\mathrm{O}_{2}$-Aufnahme wurde bei Zellsuspensionen, die mit 6 verschiedenen Röntgenstrahlendosen, 4 verschiedenen UV-Dosen oder 4 verschiedenen BPODosen behandelt worden waren, gemessen. Die Inakti- 
vierungsdosen und die beobachteten Prozentsätze Koloniebildungs-fähiger Zellen sind in Tab. 1 angegeben. Die Meßergebnisse sind in den Abb. 1 a-c zusammengefaßt und den mit unbehandelten Zellsuspensionen erzielten Kontrollwerten gegenübergestellt. Im Meßbereich zwischen 20 und $60 \mathrm{~min}$ ist ein geradliniger Anstieg aller Kurven zu verzeichnen. Die Kurven der unbehandelten Zellen zeigen je-

\begin{tabular}{|c|c|c|}
\hline $\begin{array}{c}\text { Inaktivierungs- } \\
\text { noxe }\end{array}$ & $\begin{array}{c}\text { Dosis } \\
{[\mathrm{kr}]}\end{array}$ & $\begin{array}{c}\text { o } \ddot{U} \\
\text { (makroskopische } \\
\text { Kolonien) }\end{array}$ \\
\hline $\begin{array}{l}\text { Röntgen- } \\
\text { strahlen }\end{array}$ & $\begin{array}{r}200 \\
400 \\
600 \\
800 \\
800 \\
1200 \\
1500 \\
1800 \\
1800\end{array}$ & $\begin{array}{l}2 \\
0,06 \\
0,01 \\
0,002 \\
0,0012 \\
0,001 \\
0,0003 \\
0,00014 \\
0,00012\end{array}$ \\
\hline UV & $\begin{array}{c}{\left[10^{6} \mathrm{erg} / \mathrm{cm}^{2}\right]} \\
1,5 \\
2,9 \\
4,4 \\
5,9\end{array}$ & $\begin{array}{l}0,75 \\
0,03 \\
0,002 \\
0,0008\end{array}$ \\
\hline $\begin{array}{l}\text { BPO } \\
\text { (Einwirkungs- } \\
\text { zeit } 30 \mathrm{~min} \text { ) }\end{array}$ & $\begin{array}{c}{[\%]} \\
0,4 \\
0,4 \\
0,6 \\
0,8 \\
0,8 \\
1,0 \\
1,0\end{array}$ & $\begin{array}{r}88 \\
81 \\
62 \\
20 \\
17 \\
14 \\
8\end{array}$ \\
\hline
\end{tabular}

Tab. 1. Prozentsätze überlebender Zellen $(\ddot{U})$, die zur Bildung makroskopischer Kolonien befähigt sind, nach Behandlung mit unterschiedlichen Inaktivierungsnoxen und -dosen.

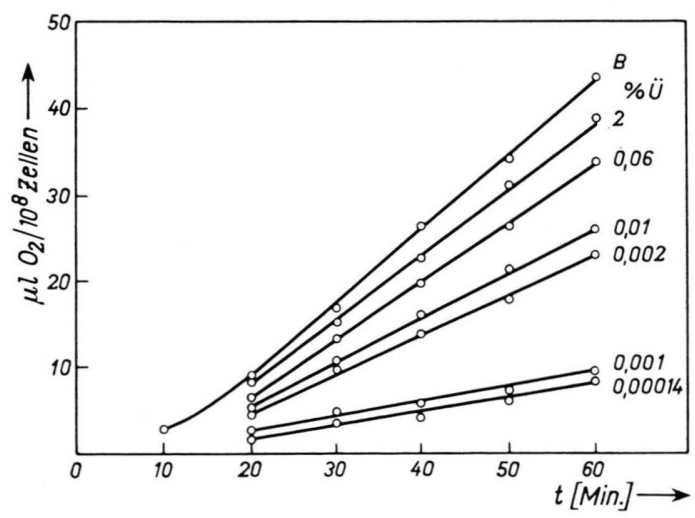

Abb. l a. Atmungsintensität unbestrahlter (B) und Röntgenstrahlen-inaktivierter Hefezellen über einen Zeitraum von $0-60 \mathrm{~min}$ nach Glucosezugabe. $\ddot{U}=$ Prozentsatz der nach Behandlung makroskopische Kolonien bildenden Zellen als $\mathrm{Ma}$ der effektiven Dosis.

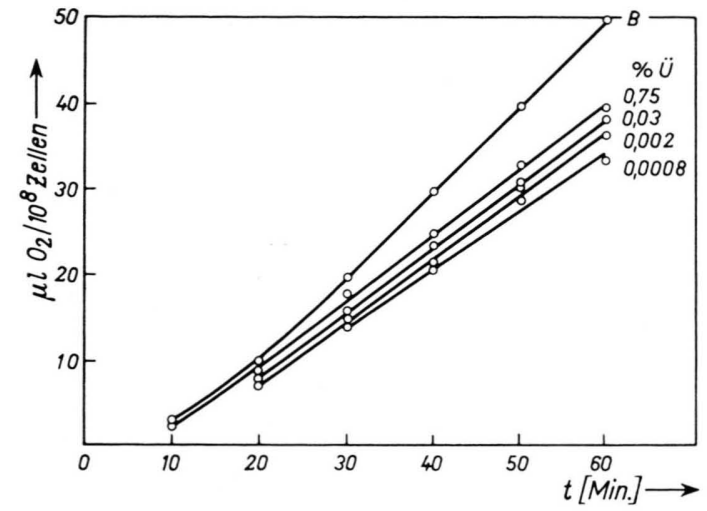

Abb. 1 b. Atmungsintensität unbestrahlter (B) und UV-inaktivierter Hefezellen.

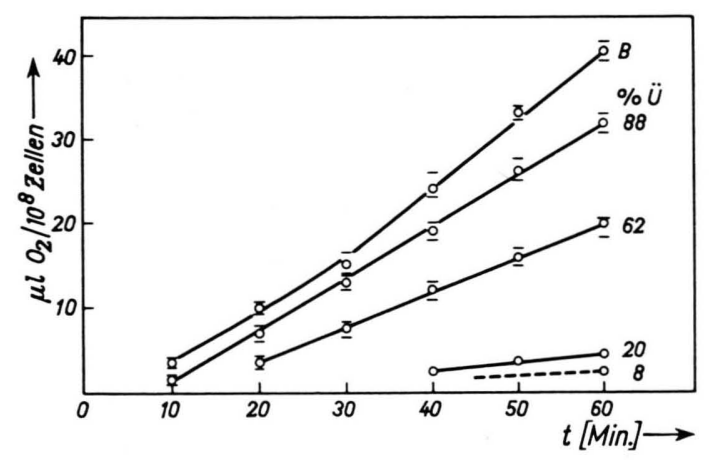

Abb. 1 c. Atmungsintensität unbehandelter (B) und BPO-inaktivierter Hefezellen.

weils den steilsten Anstieg. Mit zunehmender Inaktivierungsrate verringert sich bei den Rö- und BPOExperimenten die Kurvenneigung, die Atmungsintensität nimmt ab. Während BPO-Dosen, die zu 20\% Überlebenden, im Sinne der Bildung makroskopischer Kolonien führen, bereits die Atmung weitestgehend unterdrücken, ist nach Behandlung mit Röntgenstrahlen eine vergleichbare Reduktion der Atmungsintensität erst zu beobachten, wenn Dosen angewendet werden, die nur noch zur Bildung makroskopischer Kolonien bei weniger als $0,001 \%$ der Zellen führen. Selbst derartig hohe effektive Dosen im Sinne der Inaktivierung der Bildungsfähigkeit makroskopischer Kolonien führen nach UV-Behandlung erst zu einer relativ kleinen Reduktion der Atmungsintensität.

Die $\mathrm{CO}_{2}$-Abgabe der Zellen wurde nach Behandlung mit 3 Röntgenstrahlendosen, 1 UV-Dose oder 4 BPO-Dosen gemessen. Die Inaktivierungsdosen sind aus Tab. 1 zu ersehen. Die Meßergebnisse sind in den Abb. $2 \mathrm{a}-\mathrm{c}$ dargestellt und den Kontrollwer- 
ten unbestrahlter Zellen gegenübergestellt. Die Kurven verlaufen im gesamten Meßbereich linear. Der steilste Kurvenanstieg ist wiederum bei den Kontrollsuspensionen zu beobachten. Die Reduktion der Gärintensität tritt ähnlich wie im Falle der Reduktion der Atmungsintensität bereits bei relativ geringer Inaktivierung des Koloniebildungs-Vermögens nach BPO-Behandlung auf. Um eine gleich große

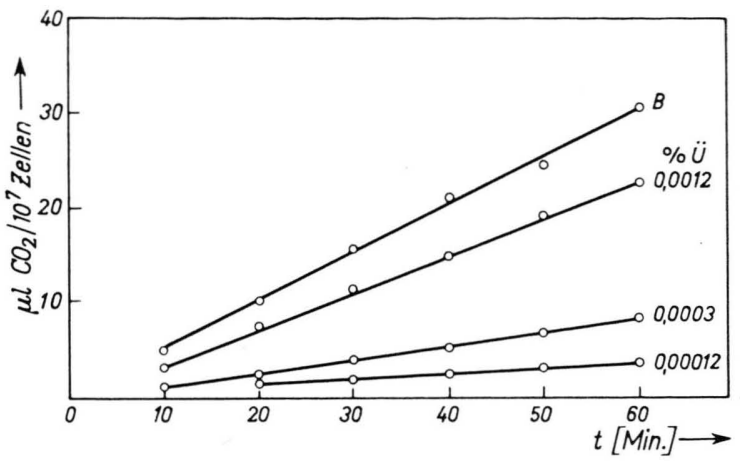

Abb. 2 a. Gärintensität unbestrahlter (B) und Röntgenstrahlen-inaktivierter Hefezellen über einen Zeitraum von 0 bis $60 \mathrm{~min}$ nach Glucosezugabe. $\ddot{U}=$ Prozentsatz der nach Behandlung makroskopische Kolonien bildenden Zellen als Maß der effektiven Dosis.

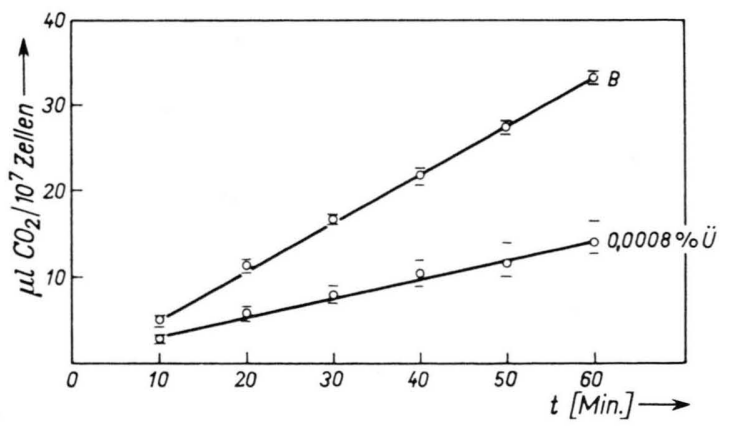

Abb. 2 b. Gärintensität unbestrahlter (B) und UV-inaktivierter Hefezellen.

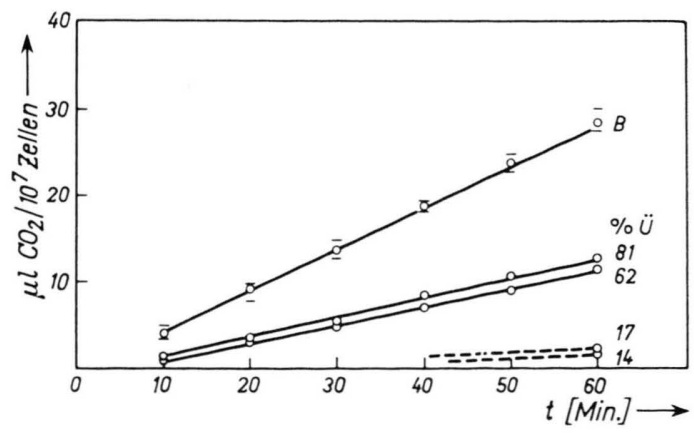

Abb. 2 c. Gärintensität unbehandelter (B) und BPO-inaktivierter Hefezellen.
Reduktion der Gärintensität nach Behandlung mit Röntgenstrahlen und UV zu erreichen, müssen Dosen appliziert werden, die das Koloniebildungs-Vermögen wesentlich stärker inaktivieren.

Um zu überprüfen, ob eine Korrelation zwischen einer Reduktion der Atmungs- und/oder Gärungsintensität einerseits und einer vollständigen Knospungshemmung andererseits vorliegt, bleibt die Knospenbildungsfähigkeit einer größeren Anzahl mit verschiedenen Dosen von Röntgenstrahlen, UV oder BPO-behandelter Zellen während einer mehrtägigen Bebrütung auf Nähragar mikroskopisch zu überprüfen. Die Ergebnisse dieser Beobachtungen sind in Abb. 3 zusammengefaßt. Jeder Kurvenpunkt resultiert aus der mikroskopischen Beobachtung der Knospenbildung von etwa 100 Zellen. Als Maß der

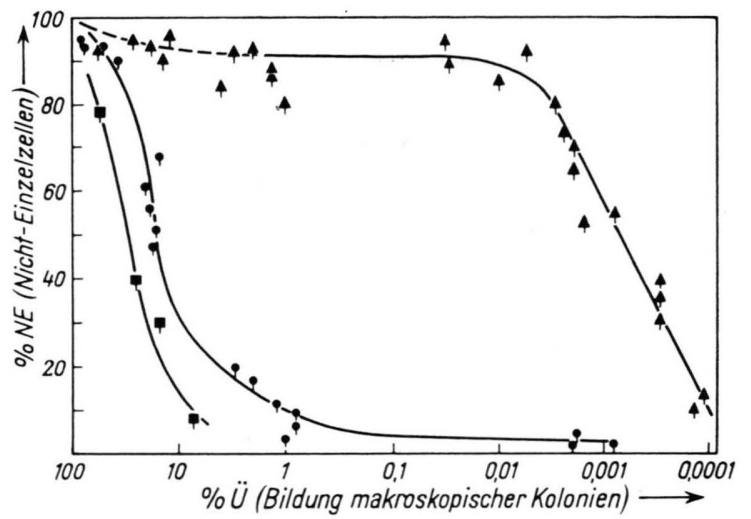

Abb. 3. Häufigkeiten der Nicht-Einzelzellen (Zellen, die wenigstens eine Knospe gebildet haben) in Abhängigkeit von Inaktivierungsnoxe und -dosis. $\dot{U}=$ Prozentsatz der nach Behandlung makroskopische Kolonien bildenden Zellen als $\mathrm{Ma} \beta$ der effektiven Dosis. $\hat{\boldsymbol{T}}=$ Röntgenstrahlen, $\boldsymbol{\uparrow}=\mathrm{UV}, \boldsymbol{\varphi}=\mathrm{BPO}$.

effektiven Dosis der 3 verwendeten Noxen wurde die Inaktivierung der Bildungsfähigkeit makroskopischer Kolonien verwendet. Die Prozentsätze derjenigen Zellen, die wenigstens eine Knospe bilden (Nicht-Einzelzellen) nehmen mit zunehmender Dosis (kleiner werdenden Prozentsätzen von $U$ ) aller 3 Noxen ab. Dabei treten charakteristische Unterschiede auf. Die Abnahme an Nicht-Einzelzellen, also die Zunahme an Einzelzellen, d. h. die Zunahme vollständig knospungsgehemmter Zellen, erfolgt nach BPO-Behandlung bereits bei relativ kleinen effektiven Dosen. Die Prozentsätze von Nicht-Einzelzellen entsprechen etwa den Prozentsätzen der Bildung makroskopischer Kolonien, d. h. nahezu alle Zellen, die nicht als Einzelzellen liegenbleiben, ent- 
wickeln sich zu makroskopischen Kolonien, es gibt so gut wie keine inaktiven Mikroklone. Auch nach UV-Behandlung nimmt der Prozentsatz an NichtEinzelzellen bereits bei relativ kleinen effektiven Dosen stark ab, wenn auch nicht so stark wie nach BPO-Inaktivierung. Inaktive Mikrokolonien von 2 Zellen bis zu einigen 100 Zellen treten mit geringer Häufigkeit auf.

Nach Röntgenstrahlenbehandlung bleibt dagegen ein restliches Knospenbildungsvermögen über einen außerordentlich großen Dosisbereich noch erhalten. Selbst bei starker Abnahme des Bildungsvermögens makroskopischer Kolonien sind die Zellen noch in der Lage, einige Tochterzellen zu bilden, und erst wenn die Bildungsfähigkeit makroskopischer Kolonien auf weniger als $0,01 \%$ herabsinkt, beginnt eine deutliche Abnahme an Nicht-Einzelzellen und damit die Zunahme einer vollständigen Knospungshemmung. Werte von etwa $10 \%$ Nicht-Einzelzellen $(90 \%$ Einzelzellen) werden nach Röntgenbestrahlung erst bei einer Dosis erreicht, die die Bildungsfähigkeit makroskopischer Kolonien auf 0,0001\% reduziert, während eine ähnliche Zunahme der Einzelzellen nach UV- oder BPO-Behandlung bereits bei effektivon Dosen auftritt, die zu 1-proz. bzw. 10-proz. Koloniebildungs-Fähigkeit führen.

\section{Diskussion}

Frühere Beobachtungen des Restteilungsmusters von Saccharomyces-Zellen, die mit verschiedenen Strahlenarten und organischen Peroxyden behandelt worden waren, hatten die Vermutung nahegelegt, daß das Auftreten einer vollständigen Knospungshemmung mit einer Störung wichtiger Stoffwechselprozesse, wie Atmung oder Gärung, korreliert sein könnte ${ }^{2}$. Ursachen für diese Vermutung lagen u. a. in der Beobachtung, daß einerseits erst sehr hohe Röntgenstrahlendosen, die die Bildungsfähigkeit makroskopischer Kolonien außerordentlich stark reduzieren, auch zu einer vollständigen Hemmung der Knospungsfähigkeit führen, andererseits Beobachtungen vorlagen, daß zur 50-proz. Hemmung der $\mathrm{CO}_{2}$-Produktion eines haploiden SaccharomycesStammes eine etwa 30-mal größere Dosis von Röntgenstrahlen notwendig ist als zur 50-proz. Hemmung der Bildungsfähigkeit makroskopischer Kolonien ${ }^{5}$. Da die Häufigkeit vollständig knospungsge-

5 E. L. Gonzales u. E. S. G. Barron, Biochim. biophysica Acta [Amsterdam] 19, 425 [1956]. hemmter Zellen in Abhängigkeit von der Häufigkeit der Ausbildung makroskopischer Kolonien für einige Inaktivierungsnoxen charakteristisch unterschiedlich ist ${ }^{2}$, bietet sich auch Gelegenheit zur Überprüfung der Frage, ob der vermuteten Korrelation eine allgemeinere Gültigkeit zukommt; ob also in Inaktivierungs-Experimenten die Prozentsätze gärungs- oder auch atmungsgehemmter Zellen aus den Anteilen vollständig knospungsgehemmter Zellen ermittelt werden können. Für einen derartigen Vergleich bieten sich als geeignete Inaktivierungsnoxen organische Peroxyde, UV- und Röntgenstrahlen an, bei denen z. B. zur Erzielung von 90\% vollständig knospungsgehemmter Zellen effektive Dosen angewendet werden müssen, die zu deutlichen Unterschieden in der Bildungsfähigkeit makroskopischer Kolonien führen $(10 \%, 1 \%$ bzw. $0,0001 \%)$.

Ein Vergleich der vollständigen Knospungshemmung sowie der Hemmung der Atmungs- und Gärungsprozesse ist aus $\mathrm{Abb} .4 \mathrm{a}$ und $\mathrm{b}$ für Röntgenstrahlen- und BPO-Behandlung bzw. UV-Behandlung zu ersehen. Die Werte für die Atmungs- und Gärungshemmungen sind ermittelt aus den 60-minWerten der Abb. 1 a bis 2 c. Die Kontrollwerte wurden jeweils gleich $100 \%$ gesetzt und die nach Behandlung erzielten Werte darauf bezogen. Es wird deutlich erkennbar, daß nach Behandlung mit Röntgenstrahlen und BPO (Abb. 4a) die drei Kurven für die Dosisabhängigkeit der Knospungs-, Atmungsund Gärungshemmung zusammenliegen, während nach UV-Behandlung (Abb. 4 b) die vollständige Knospungshemmung wesentlich früher als Gärungsund Atmungshemmung eintritt. Während der Anteil der noch zu mindestens einer Zellteilung fähigen UV-bestrahlten Zellen bereits auf etwa $5 \%$ abgesunken ist, beträgt die Atmung noch $75 \%$ der bei unbestrahlten Kontrollen erhaltenen Werte. Die Atmungsprozesse sind also wesentlich UV-resistenter als das Knospungsvermögen. Die UV-Empfindlichkeit der Gärungsprozesse wurde nur nach einer UV. Dosis untersucht, zeigte sich aber auch deutlich resistenter als das Knospungsvermögen, jedoch empfindlicher als die Atmung. Damit liegt keine Korrelation zwischen UV-induzierter vollständiger Knospungshemmung und Atmungs- oder Gärungshemmung vor.

Für eine genauere Abschätzung der Anteile atmungs- und/oder gärungsgehemmter Zellen aus Beobachtungen der Knospungshemmung scheinen jedoch auch nach einer Behandlung mit Röntgenstrah- 


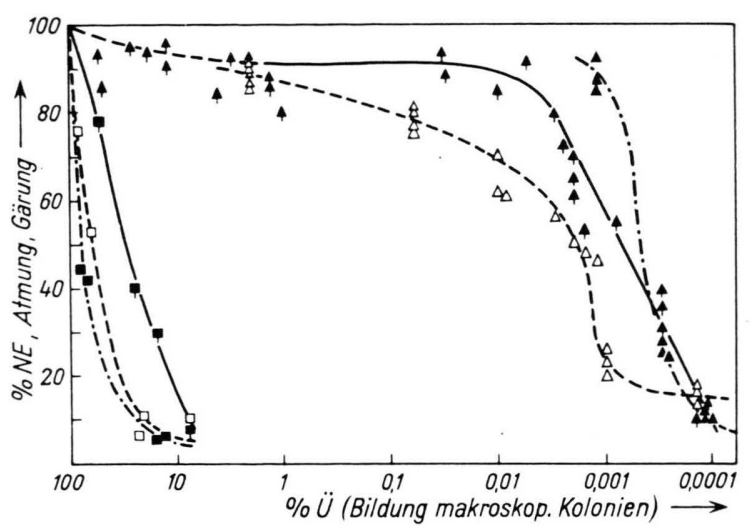

Abb. 4 a. Prozentsätze der vollständigen Knospungshemmung (gemessen als Anteil von Nicht-Einzelzellen) sowie der Hemmung von Atmung und Gärung in Abhängigkeit von der effektiven Röntgenstrahlen- und BPO-Dosis (weitere Erklärungen im Text).
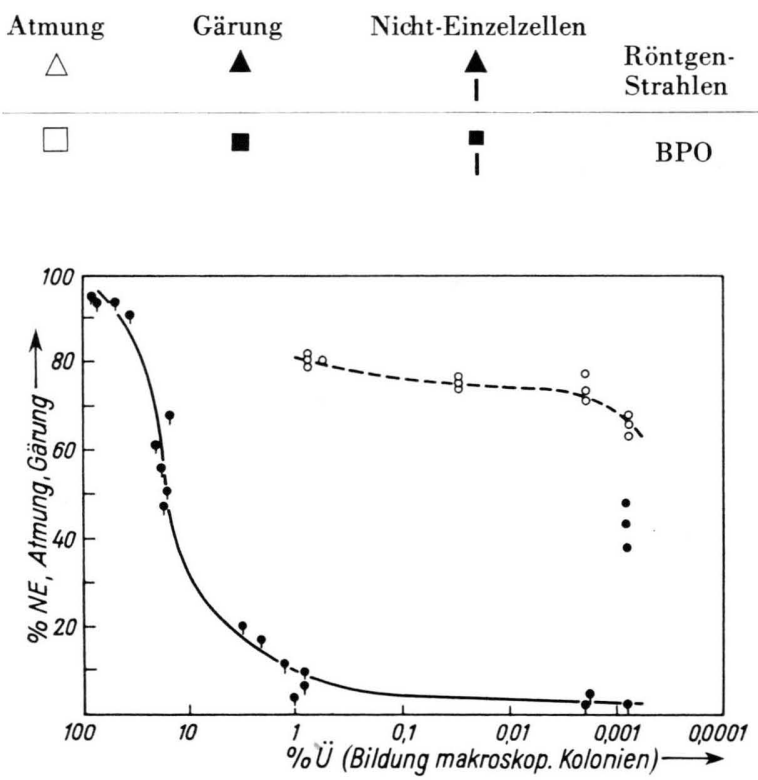

Abb. 4 b. Prozentsätze der vollständigen Knospungshemmung sowie der Hemmung von Atmung und Gärung in Abhängigkeit von der effektiven UV-Dosis.

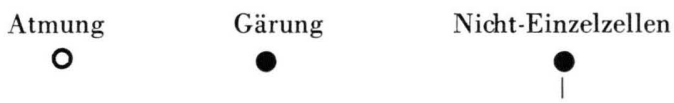

len oder BPO die Voraussetzungen zu fehlen, da die entsprechenden Kurven nicht unwesentlich voneinander abweichen. So beobachtet man z. B. nach

${ }^{6}$ H. A. Krebs u. H. L. Kornberg, Ergebn. Physiol., biol. Chem. exp. Pharmakol. 49, 212 [1957]; F. Lynen, Liebigs Ann. Chem. 546, 120 [1941] ; Naturwissenschaften 30, 398 [1942].
Röntgenbestrahlung, da $\beta$ bei 50-proz. Knospungshemmung die Atmungsintensität auf weniger als $20 \%$ herabgesunken ist, während die Gärungsintensität noch bei $80 \%$ der Kontrollen liegt. Im Falle der BPO-Inaktivierung ist bei einer 50-proz. Knospungshemmung Atmungs- und Gärungsintensität auf weniger als $20 \%$ abgesunken.

Während nach BPO-Behandlung die Atmungsund Gärungsintensität etwa in gleichem Ausmaß reduziert wird und durch eine Inaktivierung gemeinsamer Reaktionsstufen hervorgerufen sein könnte, sind diese beiden Stoffwechselprozesse sowohl nach Behandlung mit Röntgenstrahlen als auch nach UV. Behandlung unterschiedlich stark gehemmt. Die $\mathrm{O}_{2}$ Aufnahme ist wesentlich Röntgenstrahlen-empfindlicher als die $\mathrm{CO}_{2}$-Produktion. Nach UV-Bestrahlung erscheint dagegen die Gärung als strahlenempfindlicher. Aus diesen Ergebnissen folgt, daß Röntgenstrahlen und UV auf verschiedene Reaktionen innerhalb des Kohlehydratstoffwechsels wirken, daß also beide Strahlenarten den aeroben und anaeroben Kohlehydratabbau nicht durch Hemmung einer Reaktion innerhalb des gemeinsamen Abbauweges inaktivieren.

Sucht man nach Ursachen für die unterschiedliche Strahlenempfindlichkeit von Atmungs- und Gärungsprozessen, so kann man im Falle der Röntgenstrahlenfolgen an eine Hemmung der ATP-(Adenosintriphosphat) verbrauchenden Prozesse denken. Wie verschiedentlich gezeigt wurde, wird durch den Verbrauch von ATP und der damit verbundenen Freisetzung von ADP (Adenosindiphosphat) und anorganischem Phosphat die Intensität des Kohlehydratabbaues regulierend beeinflußt ${ }^{6}$. Eine Hemmung von ATP-verbrauchenden Prozessen, wie z. B. Syntheseprozessen, könnte demzufolge eine Hemmung des Kohlehydratabbaues zur Folge haben, da die notwendigen Mole ADP und anorganisches Phosphat nicht zur Verfügung stehen. Dabei sollte eine Hemmung des ATP-Verbrauchs sich auf die Atmung stärker als auf die Gärung auswirken, da bei letzterer nur 2 Mole ATP überschüssig gebildet werden, während im Ablauf der Atmungsprozesse 34 Mole ATP überschüssig freigesetzt werden ${ }^{7}$. Ein derartiges Modell würde die beobachtete relative Strahlenempfindlichkeit der Atmungs- und Gärungs-

7 H. Holzer, G. Schultz u. A. Holldorf, in: „Die Hefen“ Bd. I, Nürnberg 1960. 
H. Rister, Unterschiede in der Paarung homologer Chromosomen bei verschiedenen Cullex pipiens-Stämmen und bei Bastarden [Prophase der somatischen Reduktion] (S. 935)

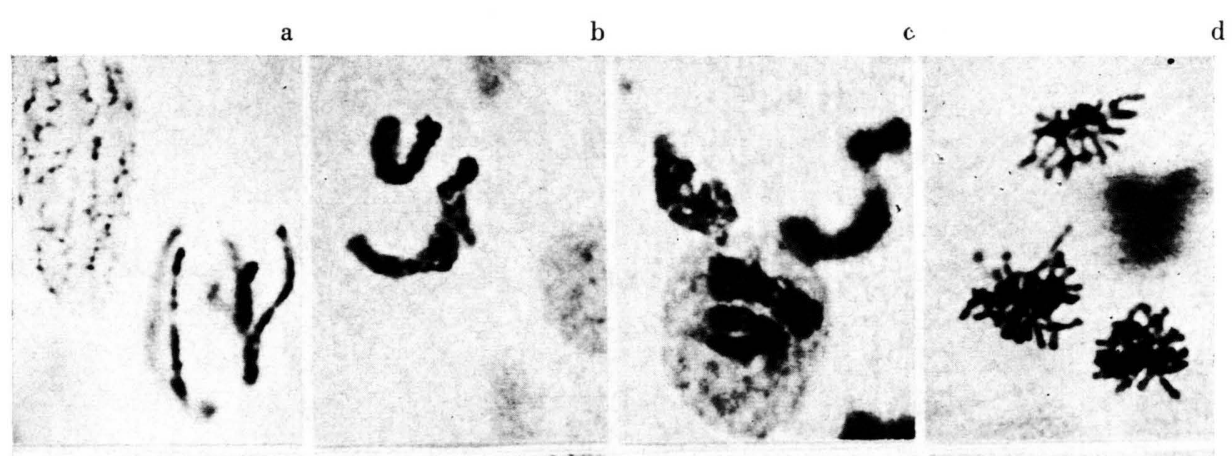

Abb. 1
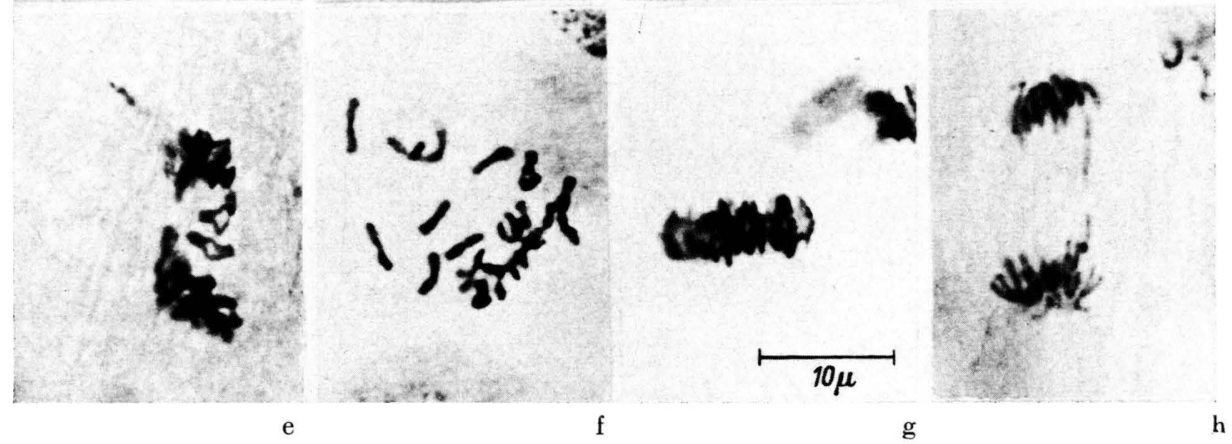

a

$\mathrm{b}$
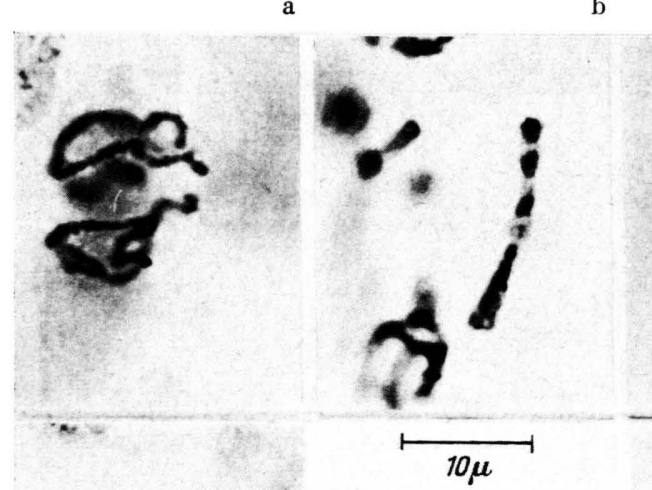

Abb. 2
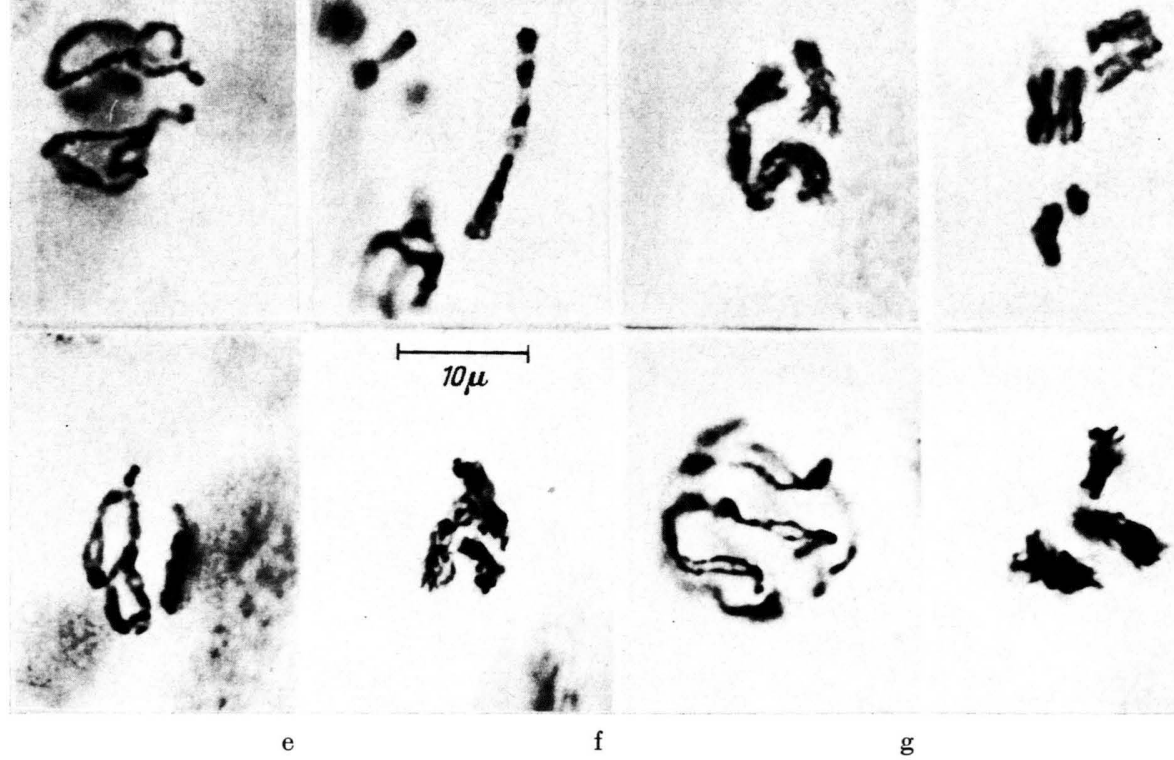

Abb. 1. Verlauf der somatischen Reduktion bei „Hamburg“ a-d Prophase; e, f Metaphase; g, h Anaphase.

Abb. 2. Prophasestadien, a-d „Scauri“; e, f „Montgomery“: $\mathrm{g}, \mathrm{h}$ „Kawasaki“. 


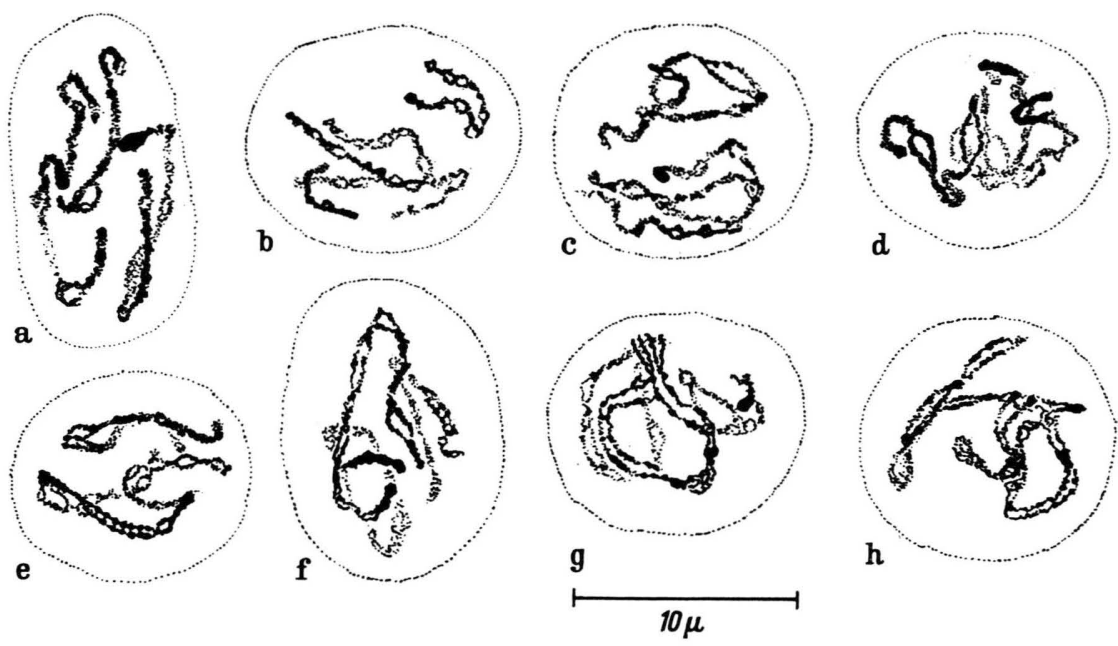

Abb. 3

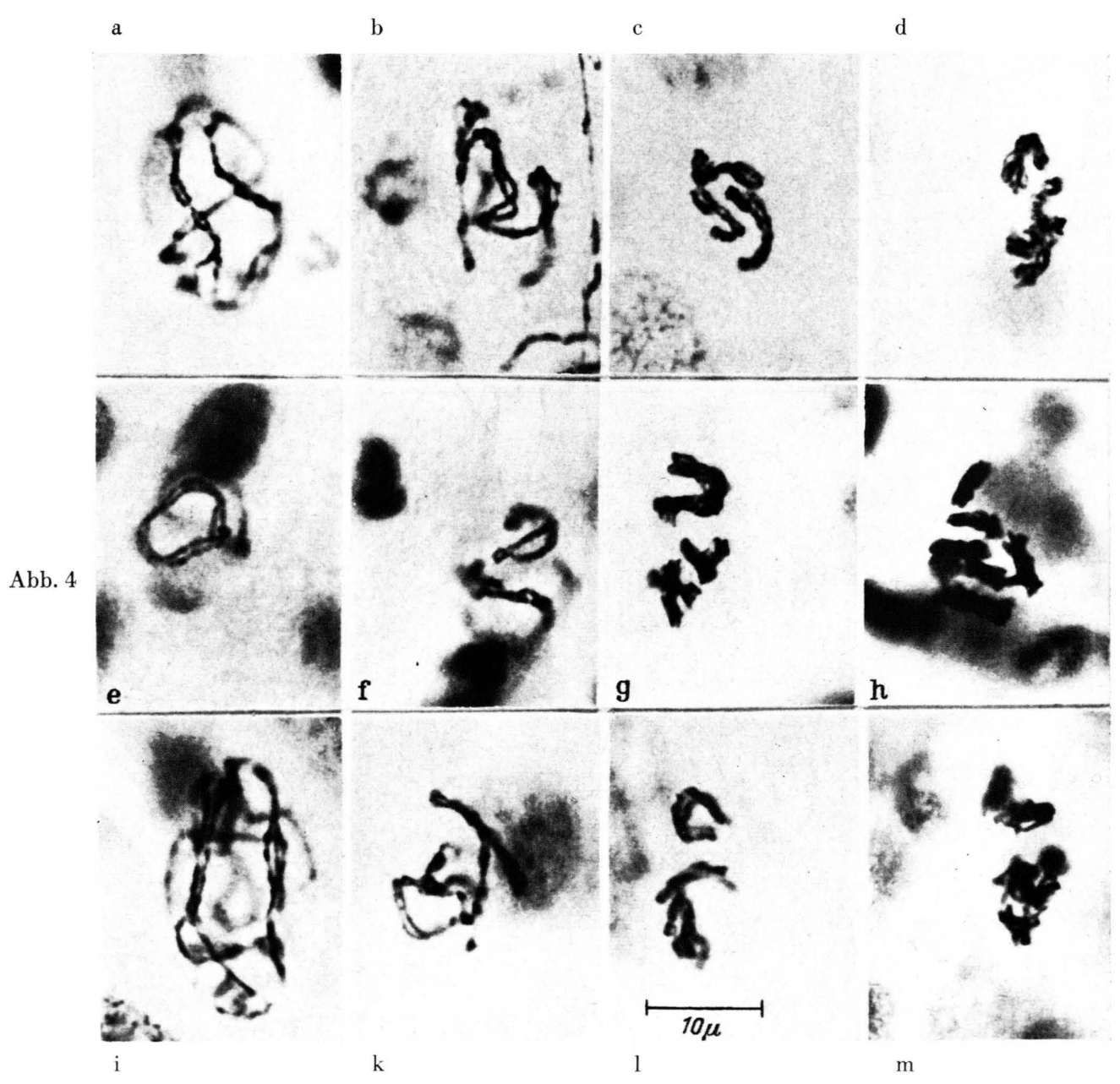

Abb. 3. Prophasestadien. a, b „Hamburg“; c "Scauri“; d „Montgomery"; e „Kawasaki"; f „Hamburg" $X$ „Scauri“; g "Hamburg" $\times$,Montgomery“; $\mathrm{h}$ "Scauri“ $\times$,Mont-

Abb. 4. Prophasestadien in Bastardpuppen. a-d „Hamburg“ gomery".

$\times{ }_{\text {,Scauri"; }} \mathrm{e}-\mathrm{h}$ "Hamburg" $\times$ "Montgomery"; $\mathrm{i}-\mathrm{m}$ "Scauri“ $\times$,Montgomery“. 
prozesse verständlich machen und einen ursächlichen Zusammenhang zwischen Knospungsprozessen als Syntheseprozessen einerseits und Atmungs- sowie Gärungsprozessen andererseits im Hinblick auf die Röntgenstrahlen-Inaktivierung aufzeigen.

$\mathrm{Ob}$ die hier behandelten Folgen der Röntgenbestrahlung tatsächlich auf eine derartige gemeinsame Ursache zurückzuführen sind, muß allerdings vorerst dahingestellt bleiben. Im Falle der UV-Bestrahlung liegen auf alle Fälle andere Verhältnisse vor. Hier scheint die relative Strahlenempfindlichkeit von Atmungs- und Gärungsprozessen gerade umgekehrt zu sein. Die Gärungsprozesse erscheinen als UV-empfindlicher als die Atmungsprozesse, und am empfindlichsten erweisen sich die Knospungsprozesse. Es kommt in einem größeren UV-Dosisbereich bereits zu einer starken Hemmung der Knospungsprozesse, während die Atmungsintensität nur geringfügig reduziert wird. Das zeigt, daß der Kohlehydratabbau auch bei Ausfall von Syntheseprozessen weitergehen kann und offensichtlich auf Grund anderer Effekte als ADP-Mangel infolge fehlender Syntheseprozesse blockiert wird.

Der Deutschen Forschungsgemeinschaft und dem Bundesministerium für wissen schaftliche Forschung danken wir für finanzielle Unterstützung.

\title{
Unterschiede in der Paarung homologer Chromosomen bei verschiedenen Culex pipiens-Stämmen und bei Bastarden (Prophase der somatischen Reduktion)* ** $^{* *}$
}

\author{
Von Helmut Risler \\ Aus dem 2. Zoologischen Institut der Johannes-Gutenberg-Universität Mainz \\ (Z. Naturforschg. 19 b, 935-937 [1964] ; eingegangen am 22. Juli 1964)
}

\begin{abstract}
Der Vergleich von Erst-Prophasen der somatischen Reduktion im Hinterdarm von Stechmückenpuppen ergibt Unterschiede in der Paarung der homologen Chromosomen.

Noch weiter aufgelockert wird diese Paarung in Bastarden verschiedener Zuchtstämme; diese Auflockerung ist bei der Kreuzung zwischen den verwandten europäischen Zuchtstämmen geringer als bei der Kreuzung mit einem amerikanischen Zuchtstamm.

Die Annahme, daß die Unterschiede im Paarungsverhalten der Chromosomen genetische Ursachen haben, wird damit gestützt.
\end{abstract}

Die Chromosomensätze der Dipteren sind dadurch gekennzeichnet, daß die homologen Chromosomen des diploiden Satzes eng gepaart sind. In den polytänen Riesenchromosomen ist diese Paarung häufig so eng, daß nur an wenigen Stellen ein Spalt zwischen ihnen sichtbar wird. Auch die Zellkerne des Hinterdarms von Stechmückenpuppen sind am Ende der Larvenentwicklung polytän (RISLER ${ }^{1}$ ). Sie treten, nachdem sie das ganze Larvenleben hindurch endomitotisch gewachsen sind, zu Beginn des Puppenstadiums in eine Mitosenperiode ein, in deren Verlauf sie in mehreren Teilungsschritten von ihrem relativ hohen Polyploidiegrad 32-64-ploid) auf

\footnotetext{
* Herrn Professor Dr. W. v. Buddenbrock $\dagger$ zum 80. Geburtstag gewidmet.

* Mit Unterstützung der Deut s chen Fors chungs gem ein s cha f t.

1 H. Risler, Biol. Zbl. 80, 413 [1961].
}

einen geringeren reduziert werden („somatic reduction“, Berger ${ }^{2 a}$ ). Berger ${ }^{2 a, b}$ und Sister M. GReLL $^{3 a, b}$ haben den Verlauf der somatischen Reduktion eingehend beschrieben. Bei eigenen Untersuchungen über diesen cytologisch wie entwicklungsgeschichtlich sehr interessanten Vorgang waren Unterschiede in den Beschreibungen der verschiedenen Autoren aufgefallen, und es wurde die Vermutung geäußert, daß sie, soweit sie sich auf die Paarung der homologen Chromosomen in der Prophase beziehen, auf der Verwendung genetisch verschiedenen Tiermaterials zurückführen lassen $\left(\right.$ RISLER $\left.^{1}\right)$. Während in den eigenen Untersuchungen die Pro-

\footnotetext{
2a Ch. A. Berger, Carnegie Inst. Publ. 496, 209 [1938].

2b Ch. A. Berger, J. Hered. 29, 361 [1938].

3a Sister Mary Grell, Genetics 31, 60 [1946].

3b Sister Mary Grell, Genetics 31, 77 [1946].
} 